\title{
SURFACE HYDROPHOBICITY MODULATES THE OPERATION OF ACTOMYOSIN- BASED DYNAMIC NANODEVICES
}

\section{SUPPLEMENTARY MATERIALS}

\section{Supplementary Figures - Captions}

Supplementary Figure 1. Chemical structures for materials used to test the modulation of motility of actin filaments: hydrophilic glass (I); hydrophobic glass (II); nitrocellulose (NC, III), Poly(methyl methacrylate) (PMMA, IV); Poly(tert-butyl methacrylate) (PtBuMA, V); and a copolymer comprising O-acryloyl acetophenone oxime copolymerized with a 4acryloyloxybenzophenone sensitizer (AAPO-ABP, VIa, VIb and VIc). Photo- and e-beam lithography can change the properties of some of the materials tested. Exposure to UV light can generate $\mathrm{COOH}$ groups on the surface of PMMA and especially PtBuMA (mechanism not shown), and $\mathrm{NH}_{2}$ groups on the surface of AAPO (structures VI a-c). The photolysed material will be however removed by development and therefore the motor protein will not be adsorbed on photolysed surfaces, but on unexposed surfaces.

Supplementary Figure 2. Definitions of velocity, acceleration and deflection angle associated with the movement of actin filaments. The large arrow represents the color-coded trajectory of the actin filament.

Supplementary Figure 3. Single histograms of velocities, accelerations and deflection angles for the four surfaces that support actomyosin motility.

Supplementary Figure 4. Length of motile filaments on different surfaces (error bars $-95 \%$ confidence). 


\section{Supplementary Figure 1.}
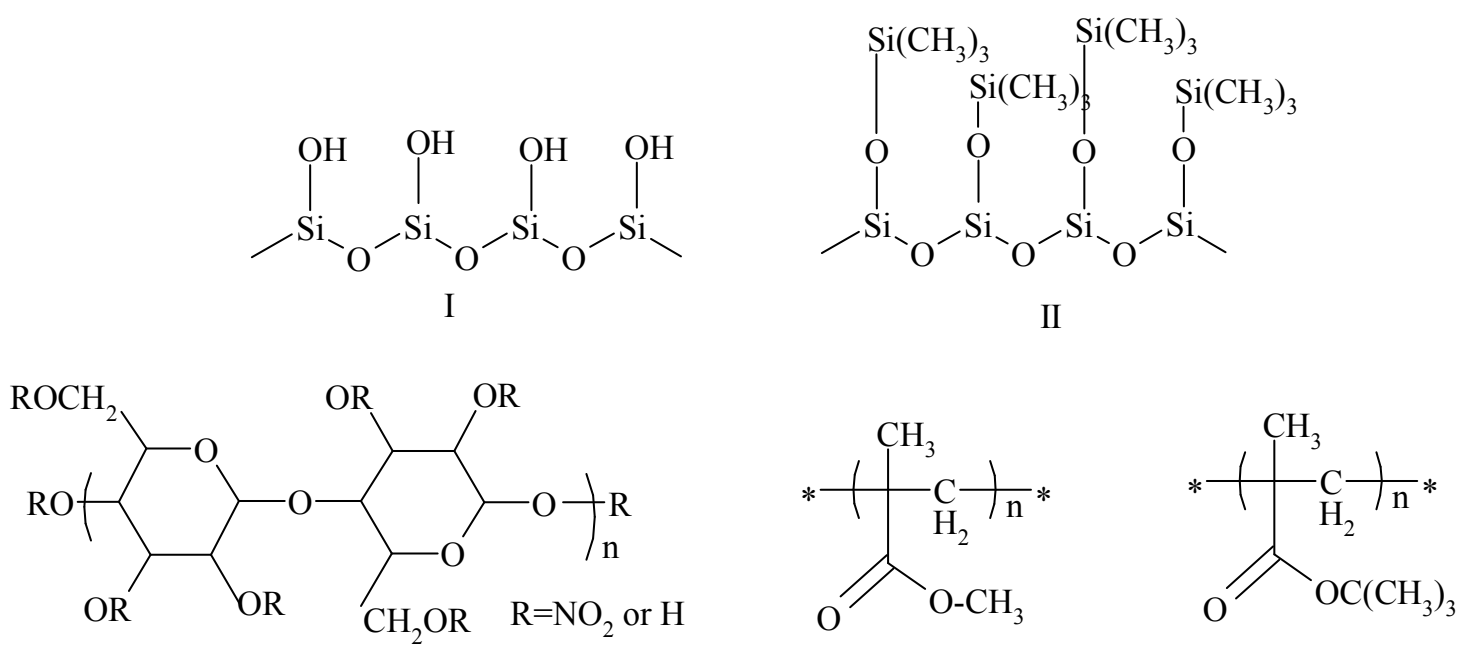

III

IV V

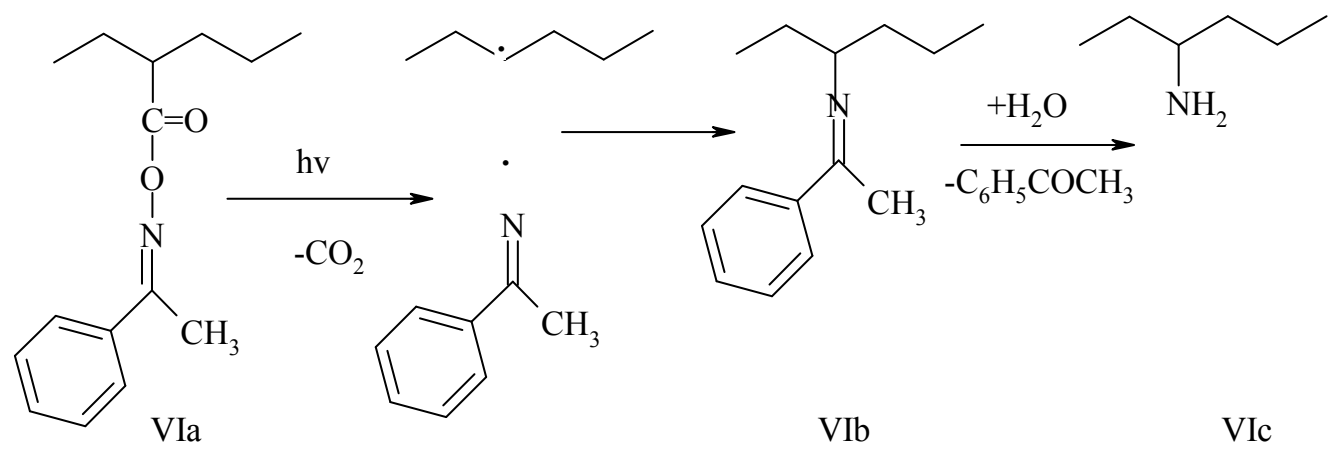


Supplementary Figure 2.

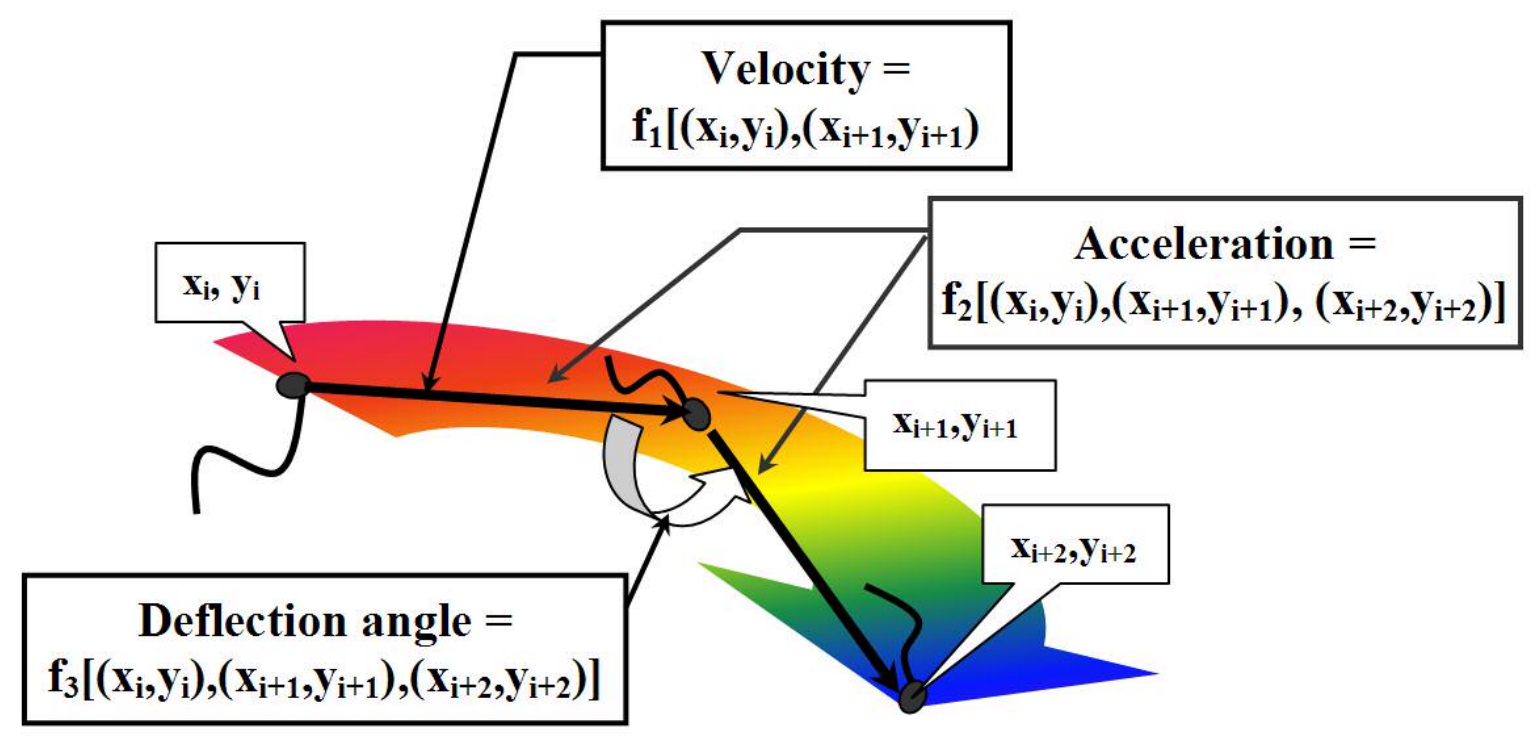


Supplementary Figure 3.
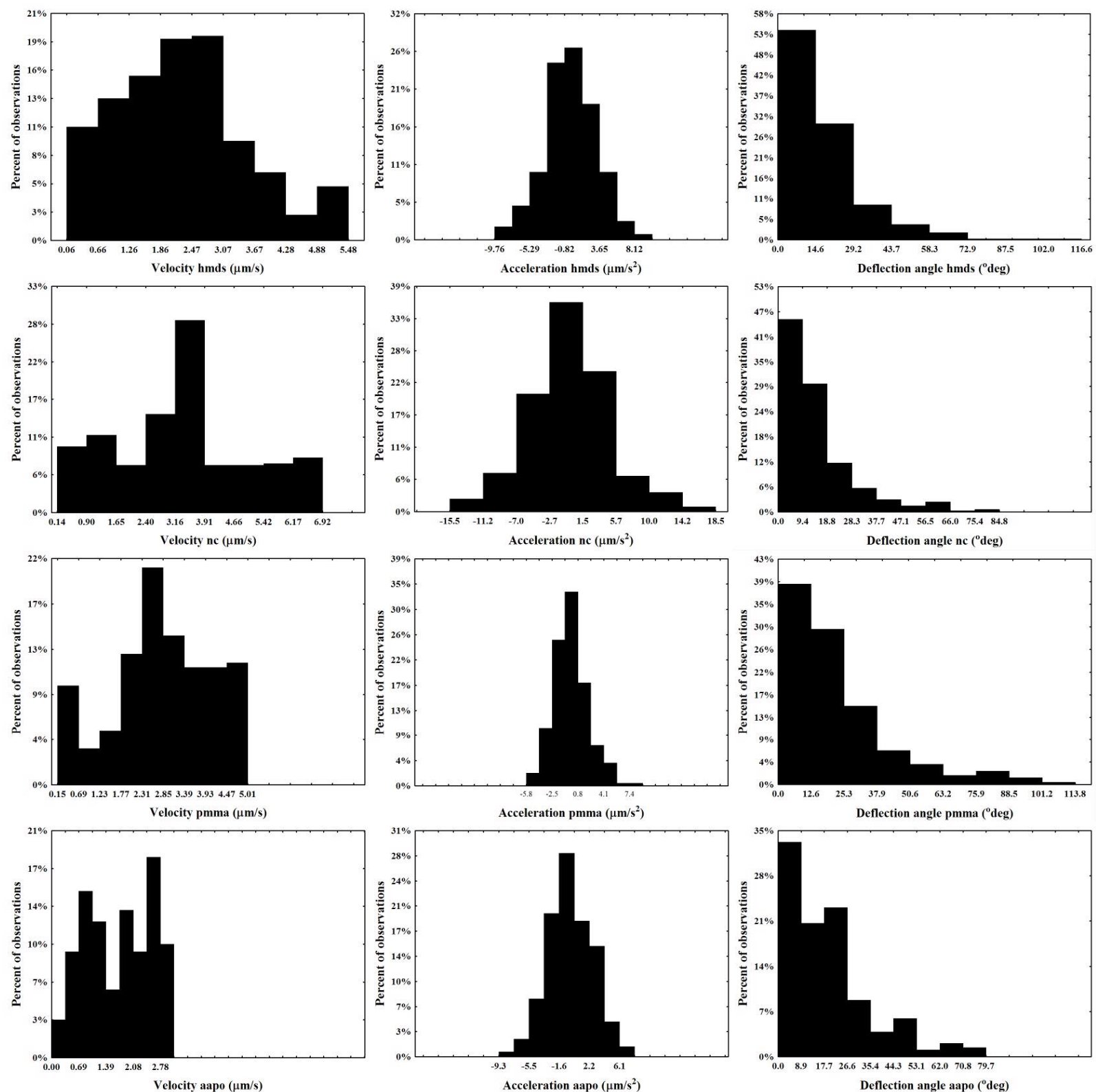
Supplementary Figure 4.

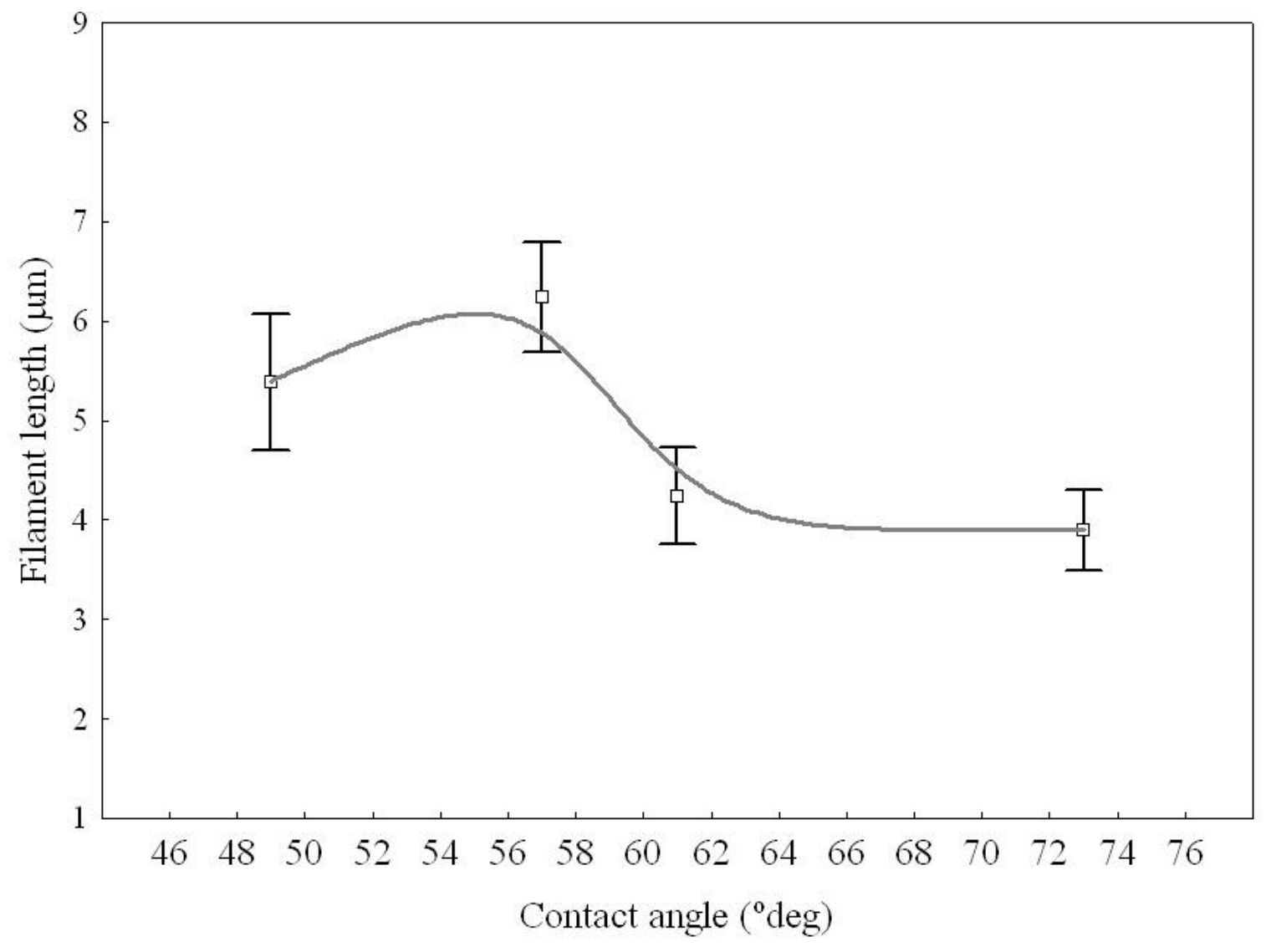




\section{Additional experimental and analysis details}

\subsection{Procedure for quantification and visualisation of molecular surface properties}

The distribution of charges and hydrophobicity on the protein molecular surfaces was computed using an upgrade of the Connolly's algorithm and visualized using DS Viewer Pro. (Accelerys Inc.). Briefly, the procedure sums the local properties (charges and hydrophobicity) of points of contact with a virtual rolling 'solvent ball' on the molecular surface. The calculated surface properties comprise: (i) global properties (i.e., total surface, total atomic charges and total aminoacid hydrophobicity); (ii) surface densities (i.e., charge density and hydrophobic density, calculated by dividing the total property to the total biomolecular area); and (iii) specific surface properties (calculated by dividing the respective property, e.g. positive charge, to the area that property turns up, e.g. positive charged area).

The use of probing balls with small radii will produce detailed molecular surface maps, while the use of probing balls with large radii, which tend to mimic flat surfaces, will result in patchy surfaces.

The use of hydrophobicities, which are aminoacid-based properties, produced low quality clustering (only 2 large clusters, for large and small proteins, respectively). The use of charges, which are atomic level properties and allow a higher resolution of the probing, produced a high quality clustering with essentially all clusters being correctly resolved. Therefore only the clustering based on atomic properties (charges) was used in the present work.

\subsection{Motility protocol}

\section{Protein Preparation}

Actin, Myosin and its sub-fragments were prepared from the back muscles of large $(2-3 \mathrm{Kg}) \mathrm{NZ}$ white rabbits.

\section{Myosin}

Myosin was prepared using a method adapted from Margossian and Lowey (1982) and is outlined in Figure 1. This method relies on a series of precipitations and sedimentations to purify the myosin by taking advantage of the differential solubility of myosin and other proteins at different ionic strengths. Crude myosin was extracted from rabbit muscle by passing the meat through a pre-chilled meat mincer twice and extracting the mince for $30 \mathrm{~min}$ in a high salt buffer. The solution is then centrifuged at $5,000 \mathrm{x} \mathrm{g}$ and the supernatant is then subjected to a series of precipitations in low salt and resuspensions in high salt. In low salt conditions $(<0.3 \mathrm{M})$, the insolubility of myosin derives from its hydrophobic rod region, whereas at a salt concentration approximating physiological conditions $(\sim 0.15 \mathrm{M})$, the myosin aggregates to form bipolar thick filaments and precipitates out of solution. As a final clarification, the myosin was centrifuged for 2 hours at 156,000x g. Purified myosin was snap frozen by placing it drop-wise into liquid nitrogen and then stored at $-80^{\circ} \mathrm{C}$ for up to 3 months. Myosin stored in this manner is prepared for use by rapidly thawing the required amount in at water bath at $30^{\circ} \mathrm{C}$.

Solution 1. Guba Straub extraction solution: $0.3 \mathrm{M} \mathrm{KCl}, 0.1 \mathrm{M} \mathrm{KPO}_{4} \mathrm{pH}$ 6.8, $2 \mathrm{mM}$ ATP, 1 $\mathrm{mM} \mathrm{MgCl} 2,0.2 \mathrm{mM}$ DTT and $0.5 \mathrm{mM}$ phenyl methyl sulfonyl fluoride (PMSF)

Solution 2. 2.4 M KCl, $20 \mathrm{mM} \mathrm{KPO}_{4} \mathrm{pH} 6.8$ and $0.2 \mathrm{mM}$ DTT. 
Remove $\sim 350$ gm of rabbit back muscle (immediately after the rabbit has been euthanized) and place on ice for $\sim 10$ min. Remove as much fat and connective tissue as possible. Mince by passing through a meat mincer.

Extract the mince in 3 vol of solution 1 for $30 \mathrm{~min}$ on ice (in the cold room).

$\downarrow$

Spin the homogenate at $6,000 \mathrm{rpm}$ for $15 \mathrm{~min}$ at $4{ }^{\circ} \mathrm{C}$.

$\downarrow$

Filter the supernatant through a fine cheese-cloth to remove any remaining fat and large lumps of muscle. Store the pellet at $-20^{\circ} \mathrm{C}$ or prepare acetone powder prep immediately.

$\downarrow$

Add 10 vol of cold water (distilled and deionised $\mathrm{H}_{2} \mathrm{O}$ ) $+0.2 \mathrm{mM}$ dithiothreitol (DTT) to the supernatant and allow the resulting precipitate to settle. I usually leave this overnight in the cold-room.

$\downarrow$

Carefully decant as much of the water without disturbing the precipitate. Spin the remainder at $6,000 \mathrm{rpm}$ for 15

$\min$ at $4{ }^{\circ} \mathrm{C}$.

$\downarrow$

Dissolve the pellet in solution 2 such that the final $[\mathrm{KCl}]$ is $0.4 \mathrm{M}$.

$\downarrow$

When completely dissolved add water to decrease the $[\mathrm{KCl}]$ from $0.4 \mathrm{M}$ to $0.28 \mathrm{M}$. and stir gently for about 15

min.

$\downarrow$

Spin $27,000 \mathrm{rpm}$ for $30 \mathrm{~min}$ at $4{ }^{\circ} \mathrm{C}$.

$\downarrow$

Add 10 vol water to supernatant and spin $6,000 \mathrm{rpm}$ for $15 \mathrm{~min}$ at $4{ }^{\circ} \mathrm{C}$.

$\downarrow$

Dissolve pellet in $0.6 \mathrm{M} \mathrm{KCl}, 10 \mathrm{mM}$ K-Pyrophosphate $\mathrm{pH}$ 7, $0.5 \mathrm{mM}$ DTT and $1 \mathrm{mM} \mathrm{NaN}_{3}$.

$\downarrow$

Spin $40,000 \mathrm{rpm}$ for $120 \mathrm{~min}$ at $4{ }^{\circ} \mathrm{C}$.

$\downarrow$

Freeze myosin (supernatant) by adding drop-wise to liquid $\mathrm{N}_{2}$ and store at $-80{ }^{\circ} \mathrm{C}$.

Figure 1: The procedure for preparing rabbit skeletal muscle myosin. All steps were carried out at $4^{\circ} \mathrm{C}$ unless otherwise stated.

\section{Heavy MeroMyosin - HMM}

HMM was prepared by a method adapted from Margossian and Lowey (1982) and is detailed in Figure 2. Briefly, myosin (either freshly prepared or from frozen stock) is adjusted to a concentration of $\sim 20 \mathrm{mg} / \mathrm{mL}$ in $0.5 \mathrm{M} \mathrm{KCl}$ and $50 \mathrm{mM} \mathrm{KPO}_{4}, \mathrm{pH} 6.5$. Once the solution has equilibrated to room temperature, the myosin is digested by addition of $\alpha$-chymotrypsin and the reaction is terminated by addition of PMSF. The solution is then dialysed in a low salt solution for $24 \mathrm{hr}$ a $4{ }^{\circ} \mathrm{C}$. Undigested myosin and the myosin rod, precipitates during the dialysis and the $\mathrm{HMM}$ is purified by centrifugation at $100,000 \mathrm{xg}$ for $60 \mathrm{~min}$ at $4{ }^{\circ} \mathrm{C}$. The concentration of HMM is then adjusted to $1 \mathrm{mg} / \mathrm{mL}$ (in $20 \mathrm{mM} \mathrm{KCl}, 10 \mathrm{mM} \mathrm{KPO}_{4} \mathrm{pH} 7.0$ and $1.5 \mathrm{mM}$ DTT) and used immediately. Alternatively, $100 \mu \mathrm{L}$ aliquots are placed into Eppendorf tubes to be snap frozen in liquid nitrogen and stored at $-80{ }^{\circ} \mathrm{C}$ for up to 3 months.

Dissolve myosin to a final concentration of $\sim 20 \mathrm{mg} / \mathrm{ml}$ in $0.5 \mathrm{M} \mathrm{KCl}$ and $50 \mathrm{mM} \mathrm{KPO}_{4} \mathrm{pH} 6.5$.

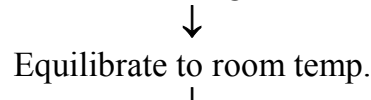

\section{$\downarrow$}

Make a stock of $3.5 \mathrm{mg} / \mathrm{ml} \alpha$-chymotrypsin in $0.001 \mathrm{~N} \mathrm{HCl}$. 
Gently stir the myosin and add $\alpha$-chymotrypsin to a final concentration of $\sim 0.07 \mathrm{mg} / \mathrm{ml}$ (ie. 1:50 dilution) incubate for $10 \mathrm{~min}$.

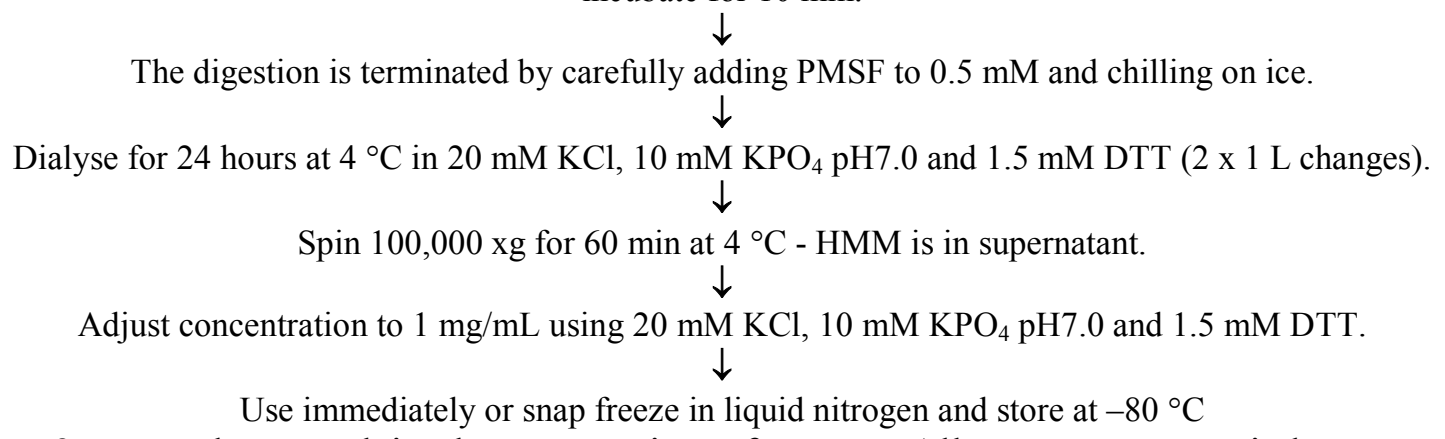

Figure 2: Procedure used in the preparation of HMM. All steps were carried out at room temperature unless otherwise stated.

\section{Labeling Actin with Rhodamine Phalloidin}

In order to visualise the actin filaments in the motility assay, they are labelled with rhodamine phalloidin (RP). Phalloidin is a seven-amino acid peptide that stabilises the actin filament by binding monomers in the middle of the filament axis. This result is achieved by effectively lowering the critical concentration of actin (defined as the concentration of actin monomers that remain unassembled under polymerising conditions).

The procedure is as described by Sellers (1993). Figure 3 outlines the procedure used in preparing an RP stock from the contents of the R-415 vial and Figure 4 details the labeling procedure. Briefly, $\sim 2 \mu \mathrm{M}$ F-actin is incubated overnight at $4^{\circ} \mathrm{C}$ with a 3 -fold excess of RP in 4 mM imidazole $\mathrm{pH} 7.0,2 \mathrm{mM} \mathrm{MgCl}_{2}, 0.1 \mathrm{mM}$ EGTA, $3 \mathrm{mM} \mathrm{NaN}_{3}$ and $1 \mathrm{mM}$ DTT (buffer A). This provides a stock of RP-actin that is stable for up to two weeks when stored on ice. RP-actin (purchased from Molecular Probes Inc. OR, \#R-415) is diluted in buffer to the desired concentration prior to commencing the assay.

Add $1.5 \mathrm{ml}$ of methanol to the entire contents of R-415 vial, such that the final concentration of RP is $6.6 \mu \mathrm{M}$.

\section{$\downarrow$}

Place $30 \mu \mathrm{l}$ aliquots into Eppendorf tubes and dry with a Speed Vac for about 3-4 hours on low heat.

\section{$\downarrow$}

Store at $-20^{\circ} \mathrm{C}$ in a light proof container

Figure 3: Procedure used for preparing RP labeling stock from R-415

Redissolve one of the RP stock tubes by adding $\sim 3 \mu$ of methanol with gentle stirring.

$\downarrow$

Add $90 \mu \mathrm{l}$ of buffer A and sonicate for about $30 \mathrm{sec}$ in a sonicating water bath.

$\downarrow$

Prepare a solution of $20 \mu \mathrm{M}$ F-actin in buffer A.

$\downarrow$

Add $10 \mu \mathrm{l}$ of the $20 \mu \mathrm{M}$ F-actin solution to the RP and gently vortex. Incubate overnight on ice.

$\downarrow$

Dilute to the desired working concentration prior to commencing the assay (usually $10-20 \mathrm{nM}$ )

Figure 4: Procedure used in the labeling of actin with RP. All steps are performed at $4{ }^{\circ} \mathrm{C}$.

Buffers and Reagents for Motility Assay (Adapted from: Sellers et al., 1993)

Buffers Used: 
A. $4 \mathrm{mM}$ Imidazole pH 7.0, $2 \mathrm{mM} \mathrm{MgCl}_{2}, 0.1 \mathrm{mM}$ EGTA, $3 \mathrm{mM} \mathrm{NaN}_{3}$ and $1 \mathrm{mM}$ DTT.

B. $0.5 \mathrm{M} \mathrm{NaCl}, 10 \mathrm{mM}$ MOPS pH 7.0, 0.1 mM EGTA and $1 \mathrm{mM}$ DTT.

C. $20 \mathrm{mM} \mathrm{KCl}, 10 \mathrm{mM}$ MOPS pH 7.2, $5 \mathrm{mM} \mathrm{MgCl}_{2}, 0.1 \mathrm{mM}$ EGTA and $10 \mathrm{mM}$ DTT.

M. $20 \mathrm{mM} \mathrm{KCl}, 10 \mathrm{mM}$ MOPS pH 7.2, $5 \mathrm{mM} \mathrm{MgCl}_{2}, 0.1 \mathrm{mM}$ EGTA, $1 \mathrm{mM} \mathrm{MgATP}$ and 10 mM DTT.

The following components are used to inhibit photobleaching: $2.5 \mathrm{mg} / \mathrm{ml}$ glucose, $0.1 \mathrm{mg} / \mathrm{mL}$ glucose oxidase (Sigma cat \# G-7016) and $0.02 \mathrm{mg} / \mathrm{mL}$ catalase (Sigma cat \# C-100). These reduce the dissolved oxygen content of the solvent and thus reduce photobleaching. Antiphotobleaching "ingredients" are added to buffer $\mathrm{M}$ just prior to commencing the assay. A stock of $50 \mathrm{mg} / \mathrm{mL}$ glucose may be kept for indefinite periods at $-20{ }^{\circ} \mathrm{C}$, but glucose oxidase and catalase (20x stocks) solutions are made immediately before commencing the assay.

\section{Basic Assay Procedure}

In general HMM (rather than whole myosin) was used for running the assays. There are several reasons for this: Firstly, it has been reported (Sellers et al., 1993) that rigor heads are "removed" when myosin is cleaved (by $\alpha$-chymotrypsin) to produce HMM and furthermore, it is possible to remove any remaining rigor heads by performing a clarifying spin with F-actin. Details of the clarifying spin are given in Figure 5. Briefly, it involves incubating HMM with equimolar amounts of F-actin and then adding Mg-ATP to dissociate "active" heads from the F-actin (rigor heads irreversibly bind to the F-actin). By centrifuging at high speed (150,000 x g) the F-actin (together with rigor heads) is pelleted and the "active" heads remain in the supernatant. This procedure works better with HMM because myosin is insoluble at the low ionic strengths required to obtain effective binding.

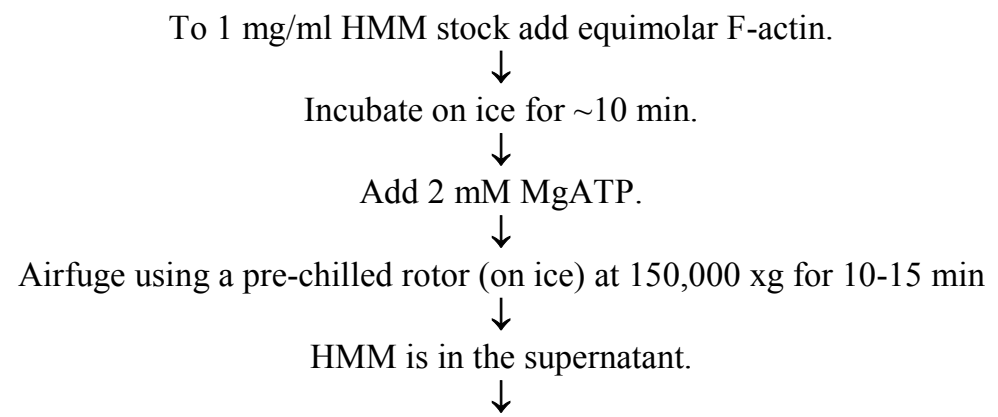

Dilute the stock HMM into buffer C to a final concentration of $0.05-0.1 \mathrm{mg} / \mathrm{mL}$ (this is kept on ice and is "reliable" for 3-4 hours).

Figure 5: Steps involved in performing a "clarifying" spin on HMM. All procedures are carried out at $4{ }^{\circ} \mathrm{C}$ unless otherwise stated.

\section{The Motility Assay}

When performing a motility assay, all solutions are kept on ice for the duration of the assay. The steps involved in the assay are detailed in Figure 6.

Introduce $2 \mathrm{vol}^{1}$ of HMM into the flow cell. Keep the slide on an angle of 20-30 (If the solutions aren't flowing freely through the chamber then a bit of filter paper at the other end helps to draw them through).

Incubate for 1-2 $\mathrm{min}$.

$\downarrow$

Wash the flow cell with 2 vol of $1 \mathrm{mg} / \mathrm{ml} \mathrm{BSA}$ in buffer C (use buffer B if using whole myosin). 
$\downarrow$
Incubate for $1-2 \mathrm{~min}$.
$\downarrow$

Flow with 2 vol of $5 \mu \mathrm{M}$ unlabelled F-actin in buffer $\mathrm{C}$ (this is usually sheared by passing through a 26 gauge needle before use).

Incubate for $2 \mathrm{~min}$.

$\downarrow$

Flow with 2-3 vol of $1.5 \mathrm{mM}$ MgATP in buffer C, immediately followed by 2-3 vol of buffer C.

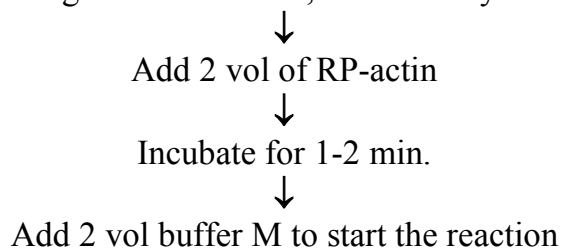

Figure 6: Details involved in performing a motility assay. All procedures are carried out in a dark room with a constant temperature of $22{ }^{\circ} \mathrm{C}$. [When using an $18 \times 18 \mathrm{~mm}$ coverslip, the volume of the motility chamber is approximately $40 \mu 1$.

\subsection{Trajectoriser}

The software for color-coding of trajectories is freely available at http://www.bionanoeng.com/downloads.html 


\section{Movies}

Movie SM1. Examples of motility movie for the surfaces that maintain motility. The placement of the motility sequences relates to the placement in Figure 4, i.e. left-bottom: AAPO; left-top: PMMA; right-top: NC; right-bottom: HMDS-glass. The representative filaments are being tracked. 